\title{
Splenorenal Collaterals as Hallmark for a Twisted Wandering Spleen in a 14-Year-Old Girl with Abdominal Pain: A Case Report
}

\author{
Rashidi Rellum ${ }^{1}$ Gerard Risseeuw ${ }^{2}$ Ivo de Blaauw ${ }^{3}$ \\ ${ }^{1}$ Department of Internal Medicine, Sint Franciscus Gasthuis, \\ Rotterdam, The Netherlands \\ 2 Department of Radiology, Ruwaard van Putten Hospital, Spijkenisse, \\ The Netherlands \\ ${ }^{3}$ Department of Pediatric Surgery, Radboud Medical Centre, \\ Nijmegen, The Netherlands \\ ${ }^{4}$ Department of Radiology, Sophia Childrens Hospital, Rotterdam, \\ The Netherlands
}

Eur J Pediatr Surg Rep 2014;2:26-28.

\author{
Maarten Lequin ${ }^{4}$
}

\begin{abstract}
Keywords

- wandering

- spleen

- torsion
\end{abstract}

Address for correspondence Rashidi Rellum, MD, Department of Internal Medicine, Sint Franciscus Gasthuis, Kleiweg 500, Rotterdam, Zuid Holland 3045 PM, The Netherlands (e-mail: rashrel@yahoo.com; rashrel84@hotmail.com).

\section{Introduction}

Wandering spleen is a known but rare entity. It may present as an acute abdominal emergency if the vascular support to the spleen is obstructed due to torsion. The presentation may also be less severe in case of incomplete vascular obstruction. We report a case of a wandering spleen in a 14-year-old girl with recurrent abdominal pain due to venous outflow obstruction resulting in developing splenorenal collaterals. In the presence of splenorenal collaterals on imaging a twisted wandering spleen should be considered, even if the spleen is in its normal position.

\section{Case Report}

A 14-year-old girl was admitted to the pediatric department of a regional hospital with a 3-week history of recurrent abdominal pain, malaise, and fatigue without fever. Physical examination revealed a palpable spleen in the normal left upper abdominal quadrant. There was no lymphadenopathy palpable. Laboratory findings were negative for Epstein Barr virus (EBV), cytomegalovirus (CMV), and hepatitis IgM; aspartate transaminase (ASAT), alanine transaminase (ALAT), carcinoembryonic antigen (CEA), CA 125, and alfa-foetoprotine were all within normal range; $\mathrm{Hb}$ was $8.0 \mathrm{mmol} / \mathrm{L}$, leucocytes $5.1 \times 10^{9} / \mathrm{L}$ with a normal differentiation and thrombocytes $108-128 \times 10^{9} / \mathrm{L}$ during hospitalization. On initial ultrasound (US), the spleen was enlarged and located in the normal left upper abdominal quadrant. The spleen had a normal architecture of the parenchyma (-Fig. 1) and a normal blood flow on color Doppler. The liver was not enlarged and had a normal architecture. The hepatic artery and portal vein showed a normal blood flow and direction on color Doppler. Furthermore, laboratory search for possible
License terms

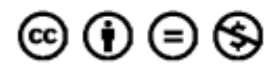

received

December 15, 2013

accepted

December 27, 2013

published online

March 28, 2014
DOI http://dx.doi.org/

10.1055/s-0034-1370776. ISSN 2194-7619. (c) 2014 Georg Thieme Verlag KG Stuttgart · New York 


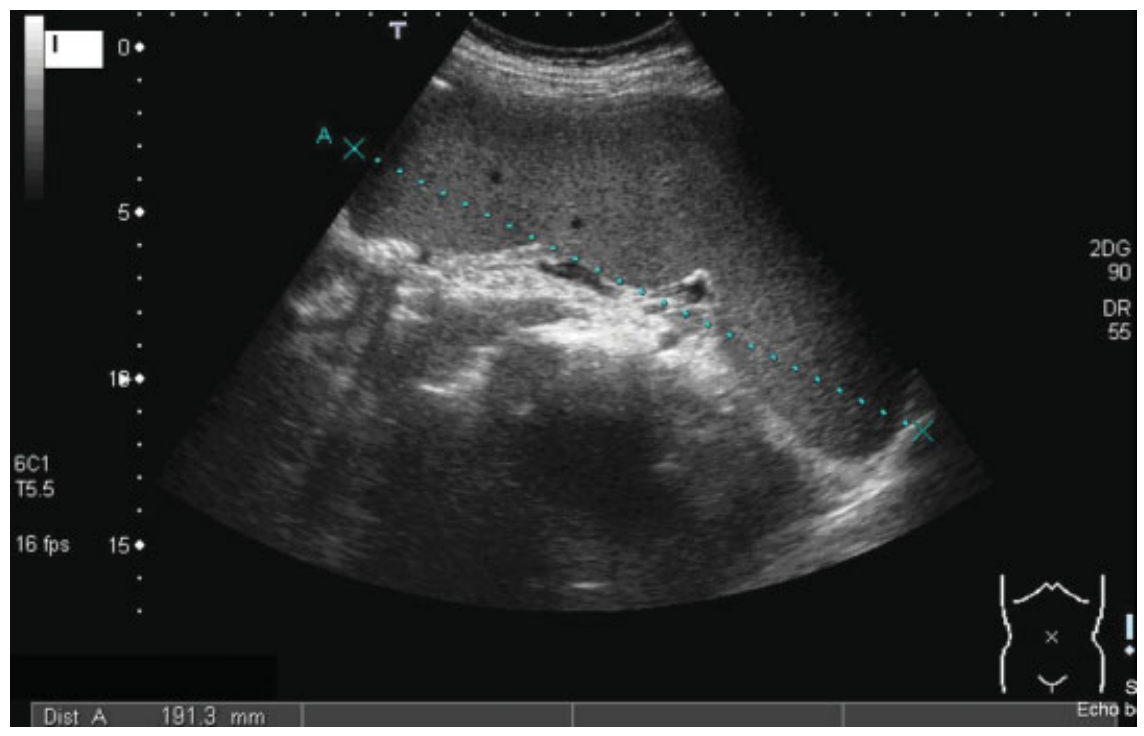

Fig. 1 Ultrasound shows a splenomegaly $(19 \mathrm{~cm})$ located in the normal left upper abdominal quadrant.

autoimmune diseases was normal. Differential diagnosis was splenomegaly e causa ignota. because there were no signs of infection, liver disease, hematologic, or autoimmune disease. As clinical symptoms progressed without further explanation, an MRI of the abdomen was made 10 days after the initial US. The MRI showed the enlarged spleen now localized in the pelvis. It also showed torsion of the splenic hilar vessels and spontaneously enlarged splenorenal collaterals (-Fig. 2), compatible with the diagnosis of a twisted wandering spleen. The patient was referred to a tertiary pediatric hospital where a pediatric surgeon was consulted. At the first elective moment, a laparoscopic splenopexy was planned.

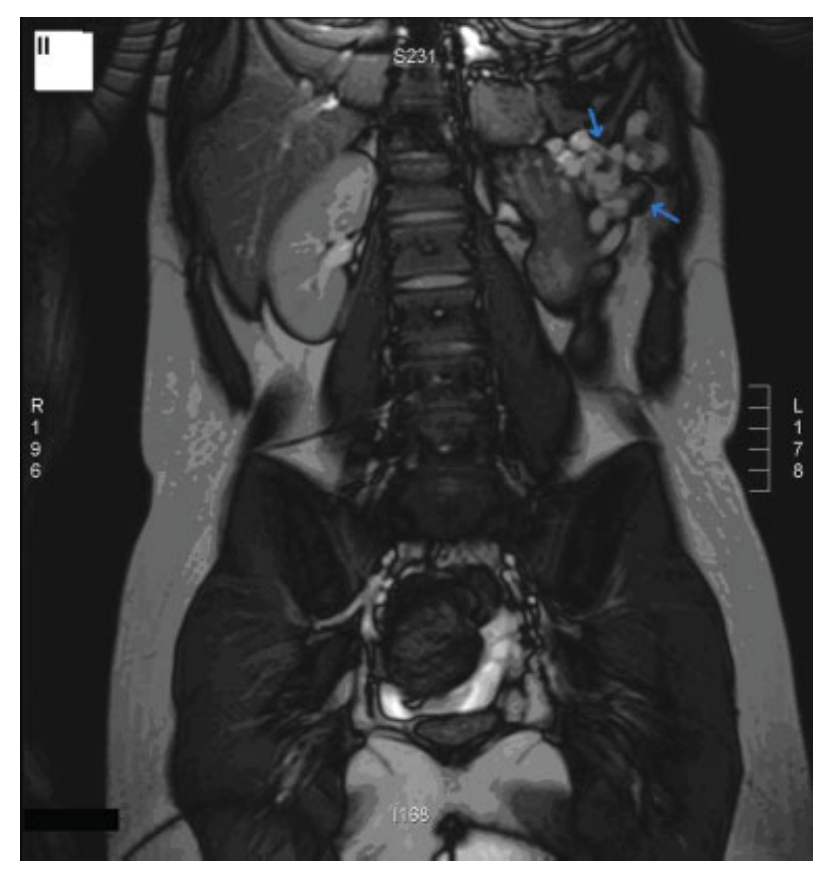

Fig. 2 Magnetic resonance imaging: the arrows show the venous escape of splenorenal collaterals at the left kidney side. The spleen is now located in the pelvic region.
The enlarged spleen was seen in the left lower quadrant. On laparoscopic view, the spleen was twisted 360 degrees clockwise. After untwisting the spleen counterclockwise, the spleen was easily brought into the left upper quadrant of the abdominal cavity. Through the $12-\mathrm{mm}$ torcart, two vicryl meshes were introduced to completely wrap the enlarged spleen. Both meshes were sutured to the left lateral abdominal wall and covered with omentum. The postoperative course was uneventful and she left the hospital 4 days after the surgery. Four weeks after surgery, she had an accident with blunt abdominal trauma. She was admitted at the emergency department and US showed a stable position of the spleen in the left upper quadrant of the abdomen. At 3 months follow-up, she was without complaints and US showed again a stable position on the left upper quadrant.

\section{Discussion}

A wandering spleen is a rare cause of abdominal pain in the pediatric population. It usually presents between the age of 20 and 40 years. ${ }^{1}$ Patients may be asymptomatic or complain of acute or chronic recurrent abdominal pain. Sometimes a mobile mass is detected in the abdomen on physical examination. The differential diagnosis of a mobile mass in the abdomen consists of a myoma, an ovarial tumor or cyst, a lymphangioma, an inflammatory pseudotumor, ${ }^{2}$ and in less than $0.2 \%$ incidence a wandering spleen. ${ }^{3}$ A wandering spleen is characterized by a mobile spleen migrating from the left upper abdominal quadrant to more caudal and pelvic regions. However, as is shown in our case, the migration is not always found on physical examination. The increased laxity of suspensory ligaments leaves the spleen solely attached to its vascular pedicle, which may result in migration of the spleen due to lengthening of the vascular pedicle. The long vascular pedicle is sensitive for acute or chronic recurrent splenic torsion. Clinically, it may result in abdominal pain. The vascularity may be hampered, resulting in 
restraining the venous return. This leads to splenic congestion and to the development of venous outflow escape, creating collaterals to the upper gastrointestinal tract and to the splenorenal area. ${ }^{4}$ In our case, splenorenal collaterals were seen on MRI, without hepatobiliary abnormalities suggestive for portal hypertension.

US is the imaging technique of choice. It is safe, rapid, and efficient to analyze the location, size, and vascularization of the spleen. Changes of its normal position, together with an abnormal splenic size correlated with the age of the patient ${ }^{5}$ and vascular obstruction with collaterals seen on color Doppler US can be seen as pathognomonic signs for a wandering spleen. A whirled appearance of the splenic vessels and the surrounding fat is suggestive for torsion of the vascular pedicle, together with ischemia due to a temporary disrupted blood flow in the hilar and parenchymal vessels. Color Doppler US allows the evaluation of patency and flow direction of the hilar and parenchymal splenic vessels. In splenic torsion, the hilar and parenchymal vessels show a low or absent amplitude of blood flow profile. Ischemia of the parenchyma is not only characterized by a change in blood flow but also by a heterogeneous echogenicity of the spleen. ${ }^{6}$

\section{Complications}

Splenorenal collaterals can rupture causing bleeding. Torsion of the spleen can be so severe that it may result in ischemia and acute splenic enlargement. This is thought to give a painful splenic capsular tension. If torsion progresses to 360 degrees, the arterial vessels become compromised thereby increasing the risk for an acute splenic infarction. Nonoperative treatment of a wandering spleen has a $65 \%$ chance of torsion with ischemic infarction of the spleen ${ }^{7}$ and therefore surgery with fixation of the spleen is generally advised.

Both congenital and an acquired conditions are described as being the cause of wandering spleens. For example, malformation or even absence of the splenic suspensory ligaments play a role in the congenital condition. ${ }^{8}$ While trauma, postdiaphragmatic hernia repair are well known acquired conditions. However, malaria, infectious mononucleosis, benign hematologic diseases, and hormonal changes are less known but have also been described as etiologic factors that could induce an acquired wandering spleen. ${ }^{8}$

Splenectomy or splenopexy is the treatment of choice. Only in case of a splenic infarction with complete necrosis or signs of malignancy a splenectomy needs to be performed. ${ }^{4}$ In most cases, a splenopexy can be done either by laparoscopy or laparotomy. ${ }^{8}$ In general, it is possible to laparoscopically derotate the spleen (if necessary) and fixate the spleen to the abdominal wall or diaphragm with resolvable meshes. Also, opening of a peritoneal pocket in the left upper abdominal wall has been suggested to reposition and fixate the spleen. ${ }^{7}$

Only in cases of severe infarction with necrosis and in cases of severe bleeding, the spleen needs to be removed.

In conclusion, signs of recurrent abdominal pain, splenomegaly, and splenorenal collaterals on imaging techniques even though the spleen is in its normal position should be suggestive for a twisted wandering spleen. Surgical intervention is necessary because of the risk for acute splenic infarction.

\section{Conflict of Interest}

None.

\section{References}

1 Desai DC, Hebra A, Davidoff AM, Schnaufer L. Wandering spleen: a challenging diagnosis. South Med J 1997;90(4):439-443

2 Sarría Octavio de Toledo L, Cozcolluela Cabrejas R, García Asensio S, Martínez-Berganza T. [Wandering spleen associated to inflammatory pseudotumor]. Radiologia 2006;48(3):173-176

3 Fujiwara T, Takehara Y, Isoda $\mathrm{H}$, et al. Torsion of the wandering spleen: CT and angiographic appearance. J Comput Assist Tomogr 1995;19(1):84-86

4 Carmona J, Lugo Vicente H. Laparoscopic splenectomy for infarcted splenoptosis in a child: a case report. Bol Asoc Med P R 2010; 102(2):47-49

5 Karmazyn B, Steinberg R, Gayer G, Grozovski S, Freud E, Kornreich L. Wandering spleen-the challenge of ultrasound diagnosis: report of 7 cases. J Clin Ultrasound 2005;33(9):433-438

6 Nemcek AA Jr, Miller FH, Fitzgerald SW. Acute torsion of a wandering spleen: diagnosis by $\mathrm{CT}$ and duplex Doppler and color flow sonography. AJR Am J Roentgenol 1991;157(2):307-309

7 Soleimani M, Mehrabi A, Kashfi A, Fonouni H, Büchler MW, Kraus TW. Surgical treatment of patients with wandering spleen: report of six cases with a review of the literature. Surg Today 2007;37(3): 261-269

8 Montenovo MI, Ahad S, Oelschlager BK. Laparoscopic splenopexy for wandering spleen: case report and review of the literature. Surg Laparosc Endosc Percutan Tech 2010;20(5): e182-e184 